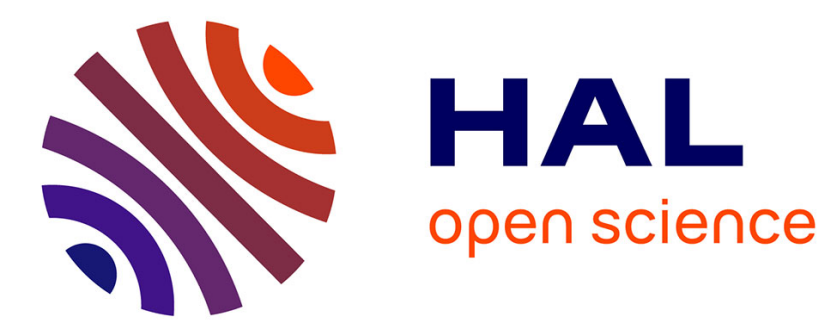

\title{
Contrôle actif des vibrations dans un système fluide/structure.
}

\author{
Bogdan Robu, Lucie Baudouin, Christophe Prieur
}

\section{To cite this version:}

Bogdan Robu, Lucie Baudouin, Christophe Prieur. Contrôle actif des vibrations dans un système fluide/structure.. Journal Européen des Systèmes Automatisés (JESA), 2011, 45 (7), pp.495-511. hal-00584310

\section{HAL Id: hal-00584310 \\ https://hal.science/hal-00584310}

Submitted on 8 Apr 2011

HAL is a multi-disciplinary open access archive for the deposit and dissemination of scientific research documents, whether they are published or not. The documents may come from teaching and research institutions in France or abroad, or from public or private research centers.
L'archive ouverte pluridisciplinaire HAL, est destinée au dépôt et à la diffusion de documents scientifiques de niveau recherche, publiés ou non, émanant des établissements d'enseignement et de recherche français ou étrangers, des laboratoires publics ou privés. 


\title{
Contrôle actif des vibrations dans un système fluide/structure
}

\author{
Bogdan Robu; Lucie Baudouin and Christophe Prieur*
}

8 avril 2011

\begin{abstract}
Résumé
Le problème du contrôle actif des vibrations induites par le ballottement du liquide dans un réservoir partiellement rempli est considéré. L'étude proposée ici, est concentrée sur l'analyse d'un dispositif expérimental imitant une aile d'avion réelle, composé d'une plaque rectangulaire en aluminium équipée d'actionneurs et de capteurs piézoélectriques fixées près du coté fixe de ladite plaque et d'un réservoir cylindrique fixé prés du coté libre de la plaque. Dans un premier temps, un modèle dimension finie du système, contenant le 5 premiers modes de vibration de la plaque et le 3 premiers modes de ballottement du liquide, est construit. Puis, en utilisant une méthode de recalage, la réponse fréquentielle est améliorée. Finalement, en utilisant la méthode par placement des pôles, un retour d'état couplé avec un observateur de type Luenberger est calculé. Des expérimentations sont ensuite réalisées pour une déformation initiale de la plaque selon le premier mode de flexion. Puis, le comportement du système sur une bande de fréquences plus large est aussi analysé.
\end{abstract}

\section{Introduction}

Les avions de future génération seront de plus en plus grands, donc de plus en plus flexibles. Pour des exemples très bien connus, nous pouvons voir les références [4] sur l'A380 Airbus ou [30] sur le concept AAW - Active Aeroelastic Wing de la NASA. De même, comme une grande partie du carburant est concentrée dans les ailes de l'avion, la masse du liquide devient une partie importante de la masse de l'aile [16]. En conséquence, la dynamique de l'aile est très influencée [27] ou [1] ce qui induit une dégradation des qualités de vol [20].

Dans ce papier nous nous intéressons principalement au couplage entre le ballottement du liquide dans le réservoir et la déformation de l'aile. Comme les quantités du liquide sont de plus en plus grandes, la fréquence propre des premiers modes de ballottement devient de plus en plus basse. Ceci a pour conséquence une interférence entre les fréquences de ballottement et les fréquences de contrôle générées par les pilotes ce qui peut poser des problèmes pour la contrôlabilité de l'engin [9]. Cette interférence va exciter de plus en plus le ballottement, jusqu'à la non-controlabilité et la destruction de l'appareil. Les références [1] et [27] peuvent être consultées pour une liste des avions qui ont eu des problèmes à cause du ballottement ou [31] pour des détailles sur la mission spatiale NEAR qui a dû être retardée à cause des mêmes raisons.

En même temps, les matériaux piézoélectriques sont de plus en plus utilisées pour mesurer et atténuer les vibrations des structures flexibles [3], [6], [8], [12], [13], [34]. Dans cet article,

*Bogdan Robu, Lucie Baudouin; CNRS ; LAAS ; 7 avenue du colonel Roche, F-31077 Toulouse, France. They are also with Université de Toulouse; UPS, INSA, INP, ISAE; LAAS; F-31077 Toulouse, France. brobu@laas.fr, baudouin@laas.fr, arzelier@laas.fr Christophe Prieur; Department of Automatic Control, Gipsa-lab, Domaine universitaire, 961 rue de la Houille Blanche, BP 46, 38402 Grenoble Cedex, France, christophe.prieur@gipsa-lab.grenoble-inp.fr. 
nous montrons comment les capteurs et actionneurs piézoélectriques peuvent être utilisées pour contrôler les vibrations d'un banc de test de type aéronautique. Par ailleurs, même si les matériaux piézoélectriques sont largement utilisées pour réduire les vibrations des structures flexibles, ils sont moins utilisées pour réduire les vibrations des structures présentant un fort couplage fluide-structure [14] et [28]. En plus, en considérant les systèmes fluide-structure pour les applications aéronautiques, les seules résultats à notre connaissance sont [17] et aussi [18] où des contrôleurs sont calculées en utilisant des méthodes numériques.

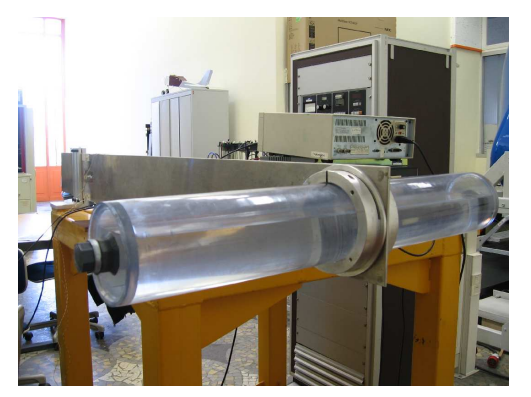

FIGURE 1 - Présentation du dispositif expérimental : plaque rectangulaire et cylindre horizontal

Le banc de test sur lequel nous travaillons est celui présenté dans la Figure 1, qui est un exemple d'un couplage fluide-structure pour des structures flexibles aéronautiques. En plus, il a été conçu pour avoir le même comportement dans les bases fréquences qu'une aile d'avion réelle [19]. Il est composé d'une plaque rectangulaire et d'un réservoir cylindrique qui peut être rempli d'eau ou de glace avec des niveaux de remplissage différents.

Dans cet article, à partir du modèle dimension infinie de la structure décrit en [23], en utilisant les équations aux dérivées partielles, nous réalisons une approximation dimension finie dans [23] et [22]. Par ailleurs, pour calculer le modèle complet, le couplage entre la plaque et le réservoir est étudié en dimension finie et infinie [22].

Le modèle dimension finie est ensuite adapté aux données expérimentales en regardant la réponse fréquentielle sur les diagrammes de Bode. Ensuite nous effectuons un recalage du modèle et nous calculons un contrôleur de type placement de pôles. L'effet du contrôleur est testé sur le système réel : d'abord pour une déformation de la plaque selon le premièr mode de flexion, puis pour une réponse fréquentielle sur la bande $[0 \cdots 50] \mathrm{Hz}$.

Le papier est organisé comme suit. Dans la partie 2, le dispositif expérimental équipée des actionneurs et capteurs piézoélectriques est d'abord présenté puis les objectifs de commande sont introduits. Dans la partie 3 , le modèle de la structure en dimension finie est présenté ainsi que le recalage du celui-ci. Puis, dans la partie 4, le contrôleur est calculé et testé sur le système réel. L'efficacité de la méthode, surtout pour la déformation de la plaque selon le premier mode, est montrée, avec les avantages et les inconvénients.

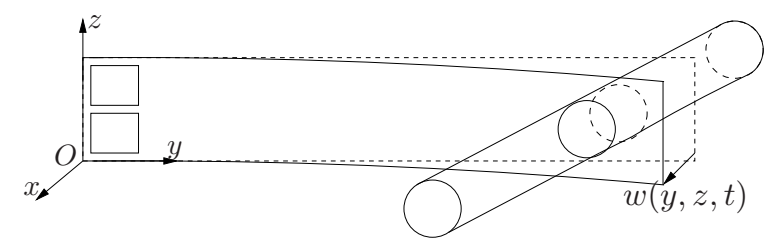

Figure 2 - Déformation de la plaque $\left(1^{\mathrm{er}}\right.$ mode) 


\section{Description du problème et du contrôle}

\subsection{Présentation du dispositif expérimental}

Le dispositif expérimental est composé d'une plaque rectangulaire en aluminium et d'un cylindre horizontal en plexiglas contenant du liquide (voir Figures 1 et 2). Il est situé à l'ISAEENSICA, Toulouse, France et il a été conçu pour avoir les mêmes fréquences de vibration qu'une aile d'avion réelle [19].

La longueur de la plaque est selon l'axe horizontal et la hauteur selon l'axe vertical. La plaque est encastrée d'un coté et libre sur les trois autres. Les caractéristiques de la plaque sont données dans le tableau 1.

\begin{tabular}{|c|c|c|}
\hline Longueur & $\mathrm{L}$ & $1.36 \mathrm{~m}$ \\
\hline Largeur & $\mathrm{l}$ & $0.16 \mathrm{~m}$ \\
\hline Epaisseur & $\mathrm{h}$ & $0.005 \mathrm{~m}$ \\
\hline Densité & $\rho$ & $2970 \mathrm{~kg} \mathrm{~m}^{-3}$ \\
\hline Module de Young & $\mathrm{Y}$ & $75 \mathrm{GPa}$ \\
\hline Coefficient de Poisson & $\nu$ & 0.33 \\
\hline
\end{tabular}

TABLE 1 - Caractéristiques de la plaque

Les actionneurs piézoélectriques sont en PZT (Lead zirconate titanate) et ils sont fixées près de l'encastrement de la plaque. Les deux capteurs sont en PVDF (Polyvinylidene fluoride) et ils sont collés sur la face opposée de la plaque par rapport aux actionneurs. Les caractéristiques des paires actionneur/capteur sont données dans le tableau 2.

\begin{tabular}{|c|c|}
\hline Longueur/largeur/épaisseur actionneur & $0.14 / 0.075 / 5 e^{-4} \mathrm{~m}$ \\
\hline Longueur/largeur/épaisseur capteur & $0.015 / 0.025 / 5 e^{-4} \mathrm{~m}$ \\
\hline Densité actionneur/capteur & $7800 \mathrm{~kg} \mathrm{~m}^{-3}$ \\
\hline Module de Young actionneur/capteur & $67 \mathrm{GPa}$ \\
\hline Coefficient piézoélectrique de l'actionneur & $-210 e^{-12} \mathrm{~m} \mathrm{~V}^{-1}$ \\
\hline Coefficient piézoélectrique du capteur & $-9.6 \mathrm{C}(\mathrm{m})^{-2}$ \\
\hline Coefficient de Poisson actionneur/capteur & 0.3 \\
\hline
\end{tabular}

TABLE 2 - Caractéristiques des pastilles piézoélectriques

Le réservoir cylindrique est centré à $1.28 \mathrm{~m}$ de la partie encastrée et il est symétrique par rapport à l'axe horizontal. A cause de la configuration du système (voir Figure 2), nous observons que le réservoir réalise un mouvement longitudinal si la plaque est en mouvement de flexion ou un mouvement de pitch si la plaque présente un mouvement de torsion. Par ailleurs, les dimensions du réservoir sont données dans le tableau 3. Il peut être rempli d'eau ou de glace jusqu'a un niveau arbitraire. S'il est rempli de glace, la modélisation est simple en considérant le liquide comme une masse fixe [25] égale à la masse du réservoir vide plus la masse de la glace. S'il est rempli du liquide mais le niveau du liquide est presque 0 ou presque égal au diamètre (dans ces cas le cylindre étant presque vide ou presque rempli du liquide), il n'y a pas de ballottement du liquide (mouvement harmonique de la surface libre du liquide) et donc le processus de modélisation est similaire à celui pour l'eau glacée.

Comme l'étude de ce dispositif expérimental a été déjà faite sans prendre en compte le ballottement, dans ce travail nous nous intéressons seulement au cas pour lesquels le niveau du liquide dans le cylindre est tel qu'un mouvement de ballottement du liquide se produit.

Le dispositif expérimental est décrit dans la Figure 3. Il est connecté au système de commande par l'intermédiaire d'un amplificateur de tension $\pm 100 \mathrm{~V}$ type PB58 de Apex Micro- 


\begin{tabular}{|c|c|}
\hline Diamètre extérieur & $0.11 \mathrm{~m}$ \\
\hline Diamètre intérieur & $0.105 \mathrm{~m}$ \\
\hline Longueur & $0.5 \mathrm{~m}$ \\
\hline Densité & $1180 \mathrm{~kg} \mathrm{~m}^{-3}$ \\
\hline Module de Young & $4.5 \mathrm{GPa}$ \\
\hline
\end{tabular}

TABLE 3 - Caractéristiques du réservoir cylindrique

technology Corporation et un amplificateur de charge 2635 de Brüer \& Kjaer. La connexion à l'ordinateur de commande se fait à l'aide d'une carte DSpaceC).

Dans un premier temps la tension générée par le correcteur (donc par la carte DSpace@) est amplifiée par l'amplificateur de tension et puis envoyée à l'actionneur piézoélectrique. La déformation de la structure est mesurée par le capteur piézoélectrique et envoyée à l'amplificateur de charges puis à la carte DSpace@.

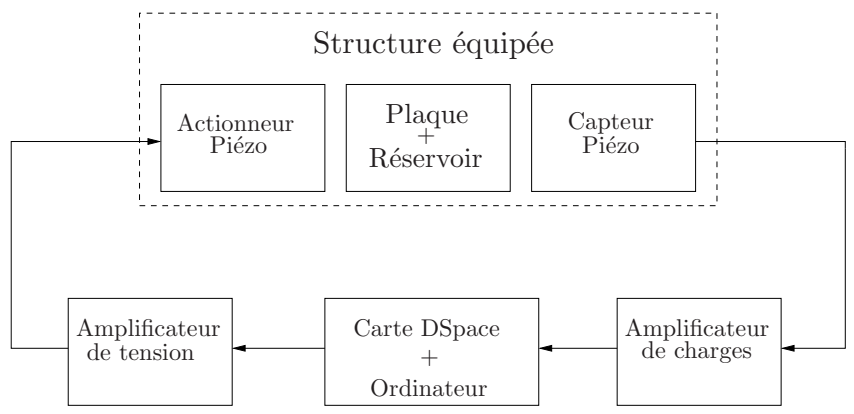

FiguRE 3 - Système expérimental

En utilisant un Analyseur de Spectre et une période d'échantillonnage de 0.004s pour la carte DSpace(C), nous pouvons visualiser la réponse fréquentielle et la réponse temporelle du système. Pour réaliser l'acquisition des données, la bibliothèque xPC Target de Matlab(c) est utilisée.

\subsection{Présentation du modèle et des objectifs de commande}

L'objectif principal du contrôle est l'atténuation des vibrations de la plaque quand la plaque est déformée selon le premier mode de vibration. La mesure de commande est la tension envoyée à un des actionneurs piézoélectriques et la mesure de sortie est la tension générée par l'amplificateur des charges connecté en sortie du capteur piézoélectrique.

Le calcul du modèle dynamique est basée sur deux équations aux dérivées partiales, une pour la plaque et l'autre pour le réservoir avec liquide. Pour pouvoir implémenter le contrôle, une approximation du modèle en dimension finie doit être réalisée. En utilisant la méthode proposée par [29] ou [22], il est démontré dans [22] que les 5 premiers modes de la plaque et 3 premiers modes du ballottement du liquide contient $95 \%$ de l'énergie du système.

Aussi, l'utilisateur doit faire attention aux tensions envoyées par le contrôleur car des très grandes amplitudes peuvent déstabiliser le système à cause de la saturation des actionneurs. Comme il a été détaillé dans la Section 2.1, les amplificateurs de tension situés en aval des actionneurs ont une limitation en amplitude égale à $\pm 100 \mathrm{~V}$.

De même, on sait que le niveau de remplissage du réservoir est variable et que le mouvement de la plaque est influencé par celui-ci. Dans notre cas, nous allons considérer un niveau de remplissage $e=0.9$ (cylindre $90 \%$ rempli du liquide). Ce niveau, proche du cas du cylindre complétement rempli du liquide, a l'avantage qu'il présente encore un phénomène de ballottement. Ce choix du niveau de remplissage, est aussi motivée par les travaux de [21] pour la 
même structure que la nôtre. D'après ces recherches, les niveaux de remplissage les plus difficiles à contrôler sont ceux pour lesquelles $e$ se rapproche de 1 (cylindre complétement rempli du liquide), qui sont aussi les plus naturellement amortis.

\section{Calcul du modèle}

\subsection{Modèle dimension finie}

La représentation d'état du dispositif expérimental est donnée par le set d'équations [1] où le vecteur d'état $X$ est une combinaison du vecteur d'état de la plaque $X_{p}$ et le vecteur d'état du liquide $X_{l}$. :

$$
X=\left(\begin{array}{c}
X_{p} \\
X_{l}
\end{array}\right) .
$$

Le vecteur d'état de la plaque est composé des paramètres cinématiques de la plaque tandis que le vecteur d'état du liquide contient les paramètres cinématiques des systèmes masse-pendule (le ballottement étant approximé par des systèmes mécaniques de type masse-pendule)

$$
\left\{\begin{array}{l}
\dot{X}=\left(\begin{array}{cc}
A_{p} & A_{l p} \\
A_{p l} & A_{l}
\end{array}\right) X+\left(\begin{array}{c}
B_{p} \\
B_{p l}
\end{array}\right) u \\
y=\left(\begin{array}{ccc}
C_{p} & 0
\end{array}\right) X .
\end{array}\right.
$$

Dans l'équation [1], les matrices liées à la plaque sont décrites par l'indice $(p)$ tandis que celles liées au ballottement du liquide par l'indice $(l)$. Par ailleurs, les indices $(p l)$ soulignent l'influence du mouvement de la plaque sur le ballottement du liquide et les indices $(l p)$ viceversa. Par conséquence, $A_{p}$ et $A_{l}$ sont les matrices dynamiques de la plaque et du liquide, pendant que $B_{p}$ et $C_{p}$ sont les matrices de commande et de sortie de la plaque. Les matrices $A_{p l}$ et $B_{p l}$ expriment l'influence de la plaque sur le liquide et la matrice $A_{l p}$ l'influence du liquide sur la plaque. La matrice $\mathbf{0}$ représente une matrice nulle de dimensions appropriées.

Par ailleurs, dans (1), $u$ est la commande du système (la tension envoyée à l'actionneur piézoélectrique) et $y$ la mesure du système (la tension envoyée par le capteur piézoélectrique).

Pour une description plus approfondie des matrices voir [23] ou [22].

Le système [1] est calculée pour $N$ modes de la plaque et pour $M \neq N$ modes de ballottement du liquide. Le calcul des valeurs de $N$ et $M$ est basé sur la contribution énergétique modale de chaque mode du système complet. Il est montré dans [11] que les premiers modes d'une structure contiennent la majorité de l'énergie de déformation de la structure. Par ailleurs, en utilisant l'expression de l'énergie proposée par [35, Chapitre 4.6] couplée avec l'approche proposée par [29] nous pouvons déterminer la contribution énergétique de chaque mode, dans l'énergie totale de la structure. Cette approche, détaillée dans [22], nous permet de trouver $N=5$ et $M=3$. Par ailleurs, il est intéressant d'observer que parmi les 5 modes de la plaque nous avons des modes de flexion mais aussi un mode de torsion. Ceci est un bon résultat car, même si nous voulons contrôler surtout les mouvements en flexion de la plaque (car ils ont la plus grande contribution énergétique [22]), nous voulons aussi étudier le comportement du contrôleur sur les mouvements de torsion de celle-ci.

A partir des valeurs de $M$ et $N$, nous calculons les dimensions des vecteurs d'état de la plaque $X_{p} \in \mathbb{R}^{10}$ et du liquide $X_{l} \in \mathbb{R}^{6}$, mais aussi du vecteur d'état du système complet $X \in \mathbb{R}^{16}$. Nous devons donc contrôler un système de dimension 16 .

\subsection{Recalage du modèle}

Le modèle mathématique du système a été validé dans [23] en faisant un test expérimental et en mesurant le temps de réponse du système réel pour atténuer une déformation initiale de la plaque. En même temps, quand on analyse la réponse fréquentielle, nous observons une 


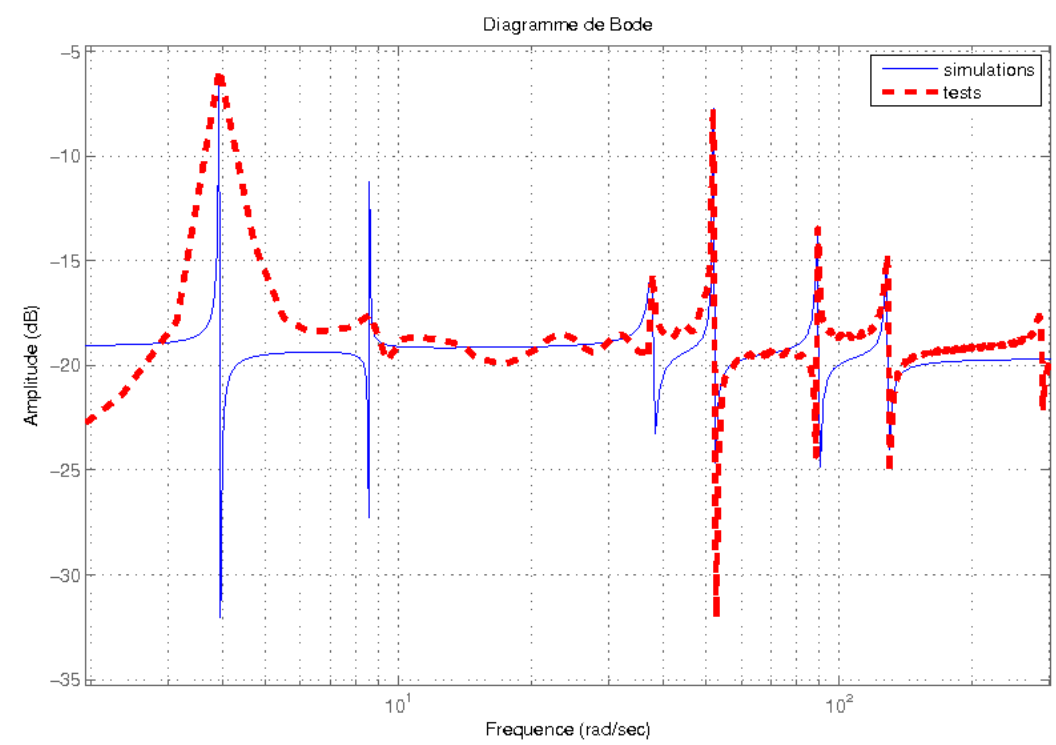

Figure 4 - Recalage du modèle pour le niveau de remplissage $e=0.9$ (modèle numérique ligne pleine et dispositif expérimental - ligne pointillée)

différence entre le réponse du modèle et celle du dispositif expérimental. C'est pour cela que des ajustements sont nécessaires quand nous analysons les diagrammes de Bode. Comme nous l'avons précédemment décrit dans [23], nous approximons dans un premier temps les fréquences propres et puis, surtout, les amplitudes des diagrammes de Bode. Pour mieux approximer le comportement en basse fréquence, un gain statique est aussi introduit.

Les résultats du recalage sont donnés dans la Figure 4. La réponse fréquentielle du modèle numérique et celle du dispositif expérimental y sont comparées. Nous observons que le recalage est assez correct sauf pour le premier mode de ballottement qui, à cause des problèmes numérique ne peut pas être changée.

Dans cette figure, le premier pic correspond au premier mode de flexion de la plaque $(0.6238 \mathrm{~Hz})$ et le deuxième pic $(1.1556 \mathrm{~Hz})$ correspond au premier mode de ballottement du liquide. Les deux autres modes de ballottement $(2.1454 \mathrm{~Hz}$ et $2.7929 \mathrm{~Hz})$ ne sont pas visibles à cause de leur très faible amplitude par rapport aux autres modes. Les quatre autres pics correspondent, dans l'ordre, au : premier mode de torsion de la plaque $(5.9977 \mathrm{~Hz})$ et aussi au deuxième $(8.2508 \mathrm{~Hz})$, troisième $(14.2495 \mathrm{~Hz})$ et quatrième $(21.0321 \mathrm{~Hz})$ mode de flexion de la plaque.

Le contrôle est réalisé en utilisant le principe de séparation. Tout d'abord nous réalisons un placement des pôles via un retour d'état. Puis nous calculons un observateur pour reconstruire les états du système.

\section{Synthèse du contrôleur et tests}

Le calcul du contrôleur par retour d'état couplé avec un observateur de type Luenberger est bien connu dans la littérature. Par example [2], [10], [33] peuvent être consultés pour une présentation générale ou [15] pour une implémentation du retour d'état au cas des structures flexibles.

A cause du fait que les états du système [1] sont totalement inconnus, un observateur identité, qui reconstruit tout l'état du système, doit être considéré avant d'implémenter le retour d'état. Pour fixer les pôles de la boucle fermée et de l'observateur, la méthode par 
placement des pôles est employée.

Par la suite, nous utilisons le chemin de commande présenté dans la Figure 5 où les matrices $K$ et $G$ doivent être calculées.

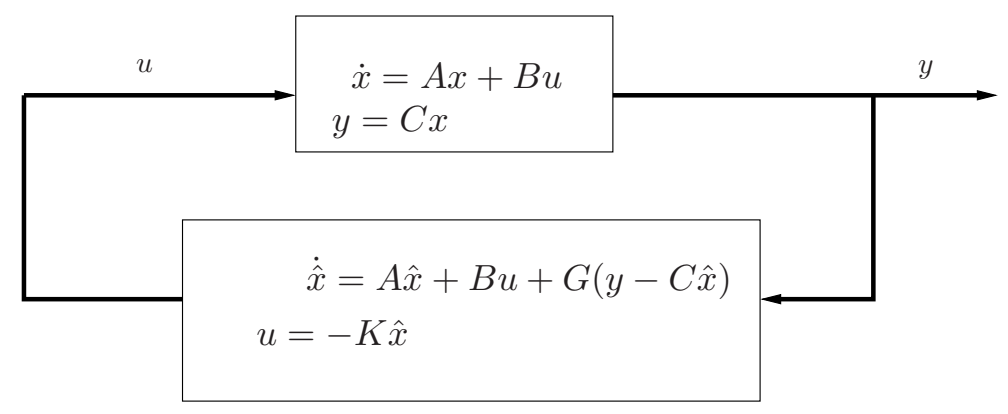

Figure 5 - Lois de commande par retour d'état couplée avec l'observateur

Dans un premier temps nous testons la commandabilité et l'observabilité du système en calculant les grammiens de commandabilité et d'observabilité, [32]. Nous observons que le système complet est commandable mai aussi observable.

A partir de cela, nous fixons la dynamique du système en boucle fermée en sélectant les valeurs propres de la matrice $A-B K$ et la dynamique de l'observateur en sélectant les valeurs propres de $A-G C$.

La sélection des pôles doit être faite avec beaucoup de soin. En général, les pôles de l'observateur doivent être plus rapides que les pôles de la boucle fermée car nous voulons que le système converge le plus vite possible vers le vrai état du système. En même temps, en respectant cette contrainte, nous sommes assurés que les performances de la boucle fermée que nous avons choisies ne sont pas trop dégradées. De même, nous avons observé que le fait d'imposer des pôles très rapides pour l'observateur, conduit à une amplification du bruit de mesure et donc à une possible excitation des hautes fréquences non prises en compte pour le calcul du modèle [7]. Ceci peut alors créer du spillover (amplification des hautes fréquences), qui peut même déstabiliser le système [5].

La même démarche doit être suivie pour choisir les pôles du système en boucle fermée. Normalement, nous voulons que ces pôles soient le plus à gauche possible, pour que le système bouclé soit plus rapide. Cette idée implique, dans un premier temps, que la tension envoyée par le contrôleur dépasse les limites de l'amplificateur de tension situé en aval de l'actionneur $( \pm 100 \mathrm{~V})$, ce qui peut avoir pour conséquence une déstabilisation du système. Dans un deuxième temps, la tension envoyée peut osciller trop vite pour contrôler le système, ce qui peut amplifier le bruit de mesure ainsi provoquer des mesures faussés.

Une solution directe à ces problèmes est de choisir des pôles plus lents mais, l'inconvénient majeur est que, le temps de réponse du système en boucle fermée sera aussi plus grand. Nous pouvons donc observer que l'utilisateur doit toujours trouver un compromis entre le temps de réponse et l'amplification du bruit de mesure.

Dans notre cas, en analysant les pôles du système en boucle ouverte nous observons 8 paires de pôles complexes conjugués ( 5 paires pour la plaque et 3 paires pour le ballottement du liquide). La position des pôles et de zéros du système en boucle ouverte est présentée dans la Figure 6. Nous pouvons facilement observer que tous les pôles ont la partie réelle négative, donc le système en boucle ouverte est stable, comme on s'attendait.

Les valeurs exactes des 8 pôles sont présentées dans le Tableau 4.

Le choix des pôles en boucle fermée est très difficile pour les raisons détaillées précédemment. Avant de trouver la meilleure solution, plusieurs jeux des valeurs ont été testé. Comme nous ne pouvons pas changer tous les pôles, car alors la tension générée par le contrôleur sera trop grande et la loi de commande sera non-applicable sur le banc de test, nous allons changer 


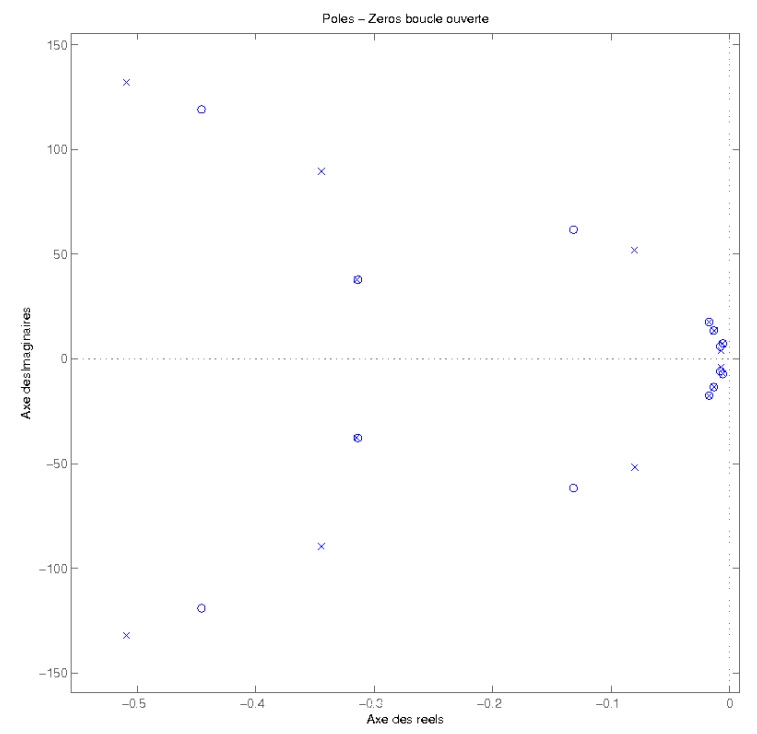

Figure 6 - Position de pôles et des zéros du système en boucle fermée $(\times$ sont les pôles, o les zéros)

seulement les valeurs des pôles dominants.

Par ailleurs, il est bien connu dans la littérature (voir [2]) que seulement la partie réelle des pôles a une influence sur le temps de réponse du système. Donc, pour faciliter l'implémentation de la loi de commande nous avons changé que la partie réelle des pôles dominants. Les valeurs des pôles que nous avons finalement obtenu sont présentées dans le Tableau 4 et elles sont comparées avec les valeurs en boucle ouverte. En ce qui concerne les valeurs des pôles de l'observateur, les références dans la littérature ([2] ou [26]) convergent sur le fait qu'ils doivent être plus rapides que les pôles en boucle fermée mais ils divergent sur le nombre de fois qu'ils doivent être plus rapides. Dans notre cas, nous avons fait un compromis entre les différentes méthodes et nous les avons choisis avec la partie réelle au moins 3 fois plus grande que la partie réelle des pôles en boucle fermée.

Le contrôleur calculé par placement des pôles est testé directement sur le dispositif expérimental. Dans un premier temps la condition initiale correspond à une déformation de $10 \mathrm{~cm}$ de la par-

\begin{tabular}{|c|c|}
\hline Pôles boucle ouverte & Pôles boucle fermée \\
\hline \hline$-0.5093 \pm 132.14 \mathrm{i}$ & $-0.5093 \pm 132.14 \mathrm{i}$ \\
\hline$-0.3447 \pm 89.53 \mathrm{i}$ & $-0.3447 \pm 89.53 \mathrm{i}$ \\
\hline$-0.0803 \pm 51.84 \mathrm{i}$ & $-0.0803 \pm 51.84 \mathrm{i}$ \\
\hline$-0.3146 \pm 37.68 \mathrm{i}$ & $-0.3146 \pm 37.68 \mathrm{i}$ \\
\hline$-0.0175 \pm 17.55 \mathrm{i}$ & $-0.0325 \pm 17.55 \mathrm{i}$ \\
\hline$-0.0135 \pm 13.48 \mathrm{i}$ & $-0.0384 \pm 13.48 \mathrm{i}$ \\
\hline$-0.0059 \pm 7.26 \mathrm{i}$ & $-0.0333 \pm 7.26 \mathrm{i}$ \\
\hline$-0.0074 \pm 3.92 \mathrm{i}$ & $-0.0324 \pm 3.92 \mathrm{i}$ \\
\hline
\end{tabular}

TABLE 4 - Valeurs des pôles du système en boucle ouverte, niveau de remplissage $e=0.9$ 
tie libre de la plaque. La réponse du système en boucle fermée, comparée à la réponse du système en boucle ouverte est présentée dans la Figure 7 pendant que la tension envoyée par le contrôleur est présentée dans la Figure 8.

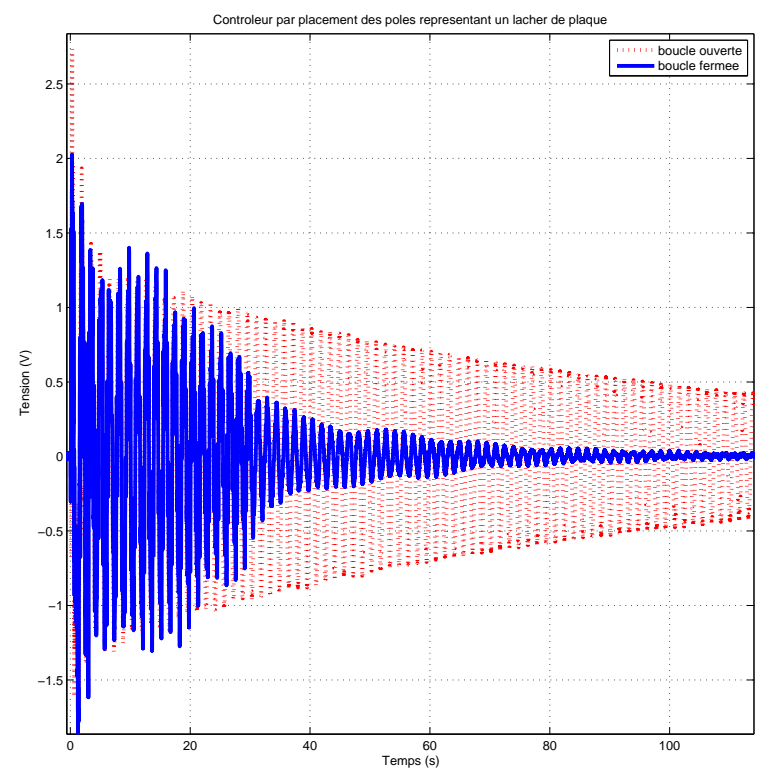

Figure 7 - Sortie du système en boucle fermée (ligne pontillée) et en boucle ouverte (ligne pleine) en utilisant un contrôleur calculé par placement des pôles, pour un niveau de remplissage $e=0.9$

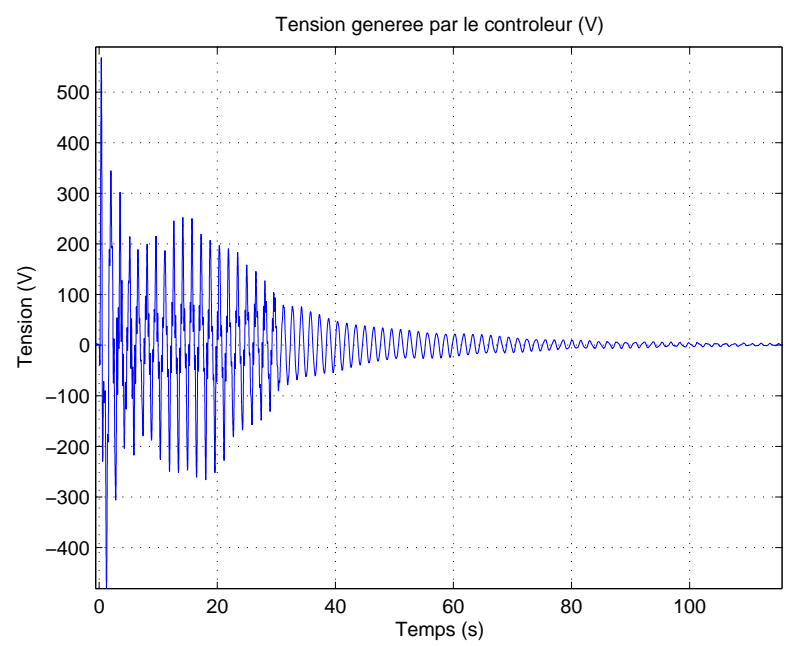

Figure 8 - Tension générée par le contrôleur

Il est très intéressant d'observer que la tension générée par le contrôleur dépasse les limites maximales de l'amplificateur de tension $\pm 100 \mathrm{~V}$. Cependant, il est logique de remarquer que la tension réelle envoyée à l'actionneur dans l'intervalle de temps $[0 \cdots 30] \mathrm{s}$ (quand la tension dépasse $100 \mathrm{~V}$ ) est en fait $+100 \mathrm{~V}$ ou $-100 \mathrm{~V}$. 
Dans un deuxième temps nous étudions aussi la réponse fréquentielle du dispositif expérimental en boucle fermée. Elle est présentée dans la Figure 9.

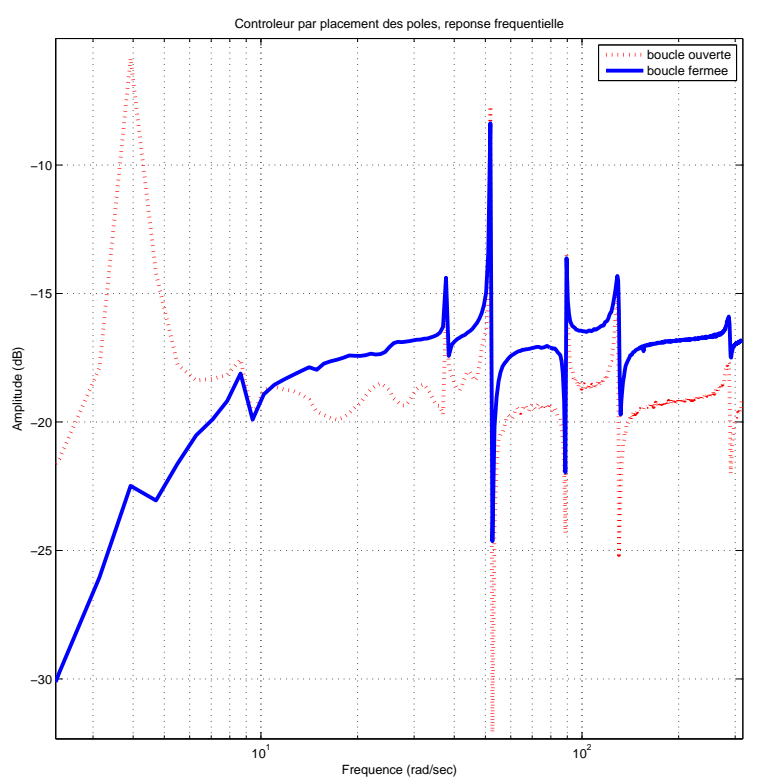

FiguRE 9 - Réponse fréquentielle du système en boucle fermée et en boucle ouverte

En analysant la Figure 9, certaines conclusions peuvent être faites. Tout d'abord, nous pouvons facilement observer que le premier mode de flexion de la plaque, celui correspondant à la première paire de pôles, est le plus atténue (une atténuation de presque $17 \mathrm{~dB}$ ). Le premier mode de ballottement (deuxième pic sur le diagramme de Bode) est aussi atténué, même s'il l'est beaucoup moins (environ $0.5 \mathrm{~dB}$ ). Ceci nous semble logique car, les pôles correspondants à ces deux pics ont été les plus déplacées par rapport à leur position en boucle ouverte. Concernant le premier mode de ballottement, sa très faible atténuation n'est pas nécessairement due à l'inefficacité de la méthode proposée (placement des pôles) mais probablement à une faible influence des actionneurs sur le ballottement. De même, nous avons vu dans [24] que les autres méthodes de calcul des contrôleurs ont aussi une très grande difficulté pour contrôler le ballottement pour ce dispositif. Concernant les autres paires de pôles qui ont été modifiées, leur présence sur le diagramme ne peut pas être observée à cause de leur très faible amplitude par rapport à celle des modes autour et à leur très faible contribution énergétique dans l'énergie totale de la plaque [22].

Nous observons aussi que le $2^{\text {eme }}$ mode de flexion de la plaque ( $4^{\text {eme }}$ pic sur les diagrammes de Bode) est aussi un peu atténué, même si la paire des pôles correspondante n'a pas été modifiée. Nous pensons que ceci est peut-être dù à l'influence des deux modes de ballottement invisibles sur la réponse fréquentielle.

De même, les pics correspondants au premier mode de torsion et aux autres modes de flexion de la plaque ont aussi leur amplitude augmentée. Ce résultat est aussi prévisible, car les pôles correspondants à ces pics n'ont pas été modifiés. A partir de cette considération, nous pouvons penser que pour une perturbation excitant des hautes fréquences, les résultats du placement des pôles seront beaucoup moins bons. 


\section{Conclusion}

Après avoir présenté l'approximation dimension finie du modèle en dimension infinie, nous avons fait un recalage du modèle en termes d'amplitude et de fréquences. Puis un contrôleur par placement des pôles est calculé pour un modèle contenant 5 modes de la plaque et 3 modes du liquide. Tests sur le dispositif expérimental sont ensuite réalisées. Dans un premier temps la condition initiale est une déformation de la plaque selon le premier mode. Par la suite, l'efficacité du contrôle est aussi vérifiée pour une réponse fréquentielle.

\section{Références}

[1] M.J. Abzug. Fuel slosh in skewed tanks. Journal of Guidance, Control and Dynamics, 19(5) :11721178, 1996.

[2] P.J. Antsaklis and A.N. Michel. Linear systems. Birkhäuser, 2006.

[3] B.P. Baillargeon and S.S. Vel. Active vibration suppression of sandwich beams using piezoelectric shear actuators : Experiments and numerical simulations. Journal of Intelligent Material Systems and Structures, 16(6):517-530, 2005.

[4] G.J. Balas. Flight control law design : An industry perspective. European Journal of Control, 9(2-3):207-226, 2003.

[5] M.J. Balas. Active control of flexible systems. Journal of Optimization Theory and Applications, 25(3) :415-436, 1978.

[6] J. Becker and W.G. Luber. Comparison of piezoelectric systems and aerodynamic systems for aircraft vibration alleviation. In Smart Structures and Materials, pages 9-18, San Diego, CA, March 1998.

[7] S.-K. Chung, J.-H. Lee, J.-S. Ko, and M.-J. Youn. A robust speed control of brushless direct drive motor using integral variable structure control with sliding mode observer. Industry Applications Society Annual Meeting and IEEE Conference Record of the 1994, 1 :393-400, 1994.

[8] K.K. Denoyer and M.K. Kwak. Dynamic modelling and vibration suppression of a swelling stucture utilizing piezoelectric sensors and actuators. Journal of Sound and Vibration, 189(1) :1331, 1996.

[9] J.J. Deyst. Effects of structural flexibility on entry vehicle control systems. Technical Report NASA SP-8016, Nasa, Space Vehicle Design Criteria, April 1969.

[10] G.C. Godwin, S.F. Graebe, and M.E. Salgado. Control system design. Prentice Hall, 2001.

[11] D. Halim and S.O.R. Moheimani. An optimization approach to optimal placement of collocated piezoelectric actuators and sensors on a thin plate. Mechatronics, 13(1):27-47, February 2003.

[12] Q. Hu and G. Ma. Variable structure control and active vibration suppression of flexible spacecraft during attitude maneuver. Journal of Aerospace Science and Technology, 9(4) :307-317, 2005.

[13] J.K. Hwang, C.-H. Choi, C.K. Song, and J.M. Lee. Robust LQG control of an all-clamped thin plate with piezoelectric actuators/sensors. IEEE/ASME Transactions on Mechatronics, 2(3) :205$212,1997$.

[14] I. Lasiecka and A. Tuffaha. Boundary feedback control in fluid-structure interactions. In Proceedings of the 47th IEEE Conference on Decision and Control, pages 203-208, Cancun, Mexico, December 2008.

[15] F. Matsuno, T. Ohno, and Y.V. Orlov. Proportional derivative and strain (dps) boundary feedback control of a flexile space structure with a closed-loop chain mechanism. Automatica, 38(7) :12011211, 2002.

[16] K.F. Merten and B.H. Stephenson. Some dynamic effects of fuel motion in simplified model tip tank on suddenly excited bending oscillations. Technical report, NASA - Langley Aeronautical Laboratory, Langley Field, VA. USA, 1952.

[17] V. Pommier-Budinger, M. Budinger, P. Lever, J. Richelot, and J. Bordeneuve-Guibé. FEM design of a piezoelectric active control structure. application to an aircraft wing model. In Proceedings of the International Conference on Noise and Vibration Engineering, Leuven, Belgium, September 2004. 
[18] V. Pommier-Budinger, Y. Janat, D. Nelson-Gruel, P. Lanusse, and A. Oustaloup. Fractional robust control with iso-damping property. In Proceedings of the American Control Conference, pages 4954-4959, Seattle, WA. USA, June 2008.

[19] V. Pommier-Budinger, J. Richelot, and J. Bordeneuve-Guibé. Active control of a structure with sloshing phenomena. In Proceedings of the IFAC Conference on Mechatronic Systems, Heidelberg, Germany, 2006.

[20] D.L. Raney, E. B. Jackson, C. S. Buttrill, and W. M. Adams. The impact of structural vibration on flying qualities of a supersonic transport. In AIAA Atmospheric Flight Mechanics Conference, Montreal, Canada, 2001.

[21] J. Richelot. Contrôle actif des structures flexibles par commande prédictive généralisée. PhD thesis, ISAE - ENSICA, 2005.

[22] B. Robu. Active vibration control of a fluid/plate system. PhD thesis, Université Paul Sabatier, Toulouse, France, 2010.

[23] B. Robu, L. Baudouin, and C. Prieur. A controlled distributed parameter model for a fluidflexible structure system : numerical simulations and experiment validations. In Proceedings of the 48th IEEE Conference on Decision and Control and 28th Chinese Control Conference, pages 5532-5537, Shanghai, P.R. China, December 2009.

[24] B. Robu, V. Budinger, L. Baudouin, C. Prieur, and D. Arzelier. Simultaneous $h_{\infty}$ vibration control of a fluid/plate system via reduced-order controller. In Proceedings of the 49th IEEE Conference on Decision and Control, Atlanta, GE. USA, December 2010.

[25] J.-S. Schotté and R. Ohayon. Various modeling levels to represent internal liquid behavior in the vibration analysis of complex structures. Journal of Computation Methods Applied Mechanics Engineering, 198 :1913-1925, 2009.

[26] A. Sinha. Linear systems, optimal and robust control. CRC Press, 2007.

[27] R.F. Stengel. Flight dynamics. Princeton University Press, 2004.

[28] T. Terasawa, C. Sakai, H. Ohmori, and A. Sano. Adaptive identification of MR damper for vibration control. In Proceedings of the 43rd IEEE Conference on Decision and Control, pages 2297-2303, Atlantis, Bahamas, December 2004.

[29] S. Tliba and H. Abou-Kandil. $H_{\infty}$ control design for active vibration damping of flexible structure using piezoelectric transducers. In Proceedings of the 4th IFAC Symposium on Robust Control Design, Milan, Italy, June 2003.

[30] D. Voracek, E. Pendleton, E. Reichenbach, K. Griffin, and L. Welch. The active aeroelastic wing phase I flight research through January 2003. Technical Report NASA TM-210741, Nasa, Dryden Flight Research Center, Edwards, CA. USA, April 2003.

[31] J.P.B. Vreeburg and D.J. Chato. Models for liquid impact onboard Sloshsat FLEVO. In Proceedings of the AIAA Space Conference, Long Beach, CA. USA, 2000.

[32] L.C. Westphal. Handbook of control system engineering, 2nd edition. Kluwer Academic Publisher, 2001.

[33] L.A. Zadeh and C.A. Desoer. Linear system theory, the state space approach. McGraw Hill Book Company, New York, 1963.

[34] J. Zhang, L. He, E. Wang, and R. Gao. Active vibration control of flexible structures using piezoelectric materials. In Proceedings of the 2009 International Conference on Advanced Computer Control, pages 540-545, Shenyang, P.R. China, January 2009.

[35] K. Zhou, J.C. Doyle, and K. Glover. Robust and optimal control. Prentice Hall, N.J., 1996. 